\title{
A preliminary inquiry into the aggregation of compositional data
}

\author{
Raúl Pérez-Fernández ${ }^{a, b}$ and Bernard De Baets ${ }^{a}$ \\ ${ }^{a}$ KERMIT, Department of Data Analysis and Mathematical Modelling, Ghent University, \\ Coupure links 653, 9000 Gent, Belgium, \\ email: raul.perezfernandez@ugent.be; bernard.debaets@ugent.be \\ ${ }^{b}$ Department of Statistics and O.R. and Mathematics Didactics, University of Oviedo, Spain, \\ e-mail: perezfernandez@uniovi.es
}

\begin{abstract}
The aggregation of compositional data vectors arises naturally in many fields of application. In particular, the weighted centroid is inherently linked to the process of mixing two or more compounds. However, as natural and simple as this process may be, the weighted centroid does not fit the standard definition of an aggregation function since there is no order relation, nor associated monotonicity, in the setting of compositional data. In this conference paper, we prove that the weighted centroid fits within the framework of penalty-based aggregation (which does not build upon the property of monotonicity) and discuss how all convex hull internal functions for the aggregation of multidimensional data could potentially be used for the aggregation of compositional data. Interestingly, the weighted centroid is indeed monotone when seen as a convex hull internal function for the aggregation of multidimensional data.
\end{abstract}

Keywords: Aggregation, Compositional data, Penalty functions, Weighted centroid.

\section{Introduction}

Penalty functions are a common tool in data aggregation $[6,7,20]$. Intuitively, a penalty function measures the disagreement of a list of objects with a single object. Most existing aggregation processes coming from many different fields - such as the method of Kemeny [14] in social choice theory, the median procedure [3] in relational calculus and the search for the closest string [16] in computer science - can be seen as processes aiming at minimizing some appropriate penalty function given the list of objects to be aggregated [18].
Fields such as soil science, chemistry, biology and environmental science make daily use of some basic techniques for the aggregation of compositional data. In particular, whenever two or more compounds are mixed (with a known mixing ratio), the composition of the obtained construct is known to be given by the weighted centroid of the compositions of the original compounds. This simple function for the aggregation of compositional data, which has been overly-studied in the field of statistics, can also be seen as a process aiming at minimizing some appropriate penalty function given the compositions of the different compounds. In this paper, we will position this function within the framework of penalty-based data aggregation and we will discuss some alternatives that - although they might lack a natural physical interpretation - could be used for the aggregation of compositional data.

The remainder of the paper is structured as follows. Section 2 is devoted to recalling some preliminary notions in the field of aggregation theory. In Section 3, we introduce the weighted centroid for compositional data and illustrate how it is linked to a mixture process. The weighted centroid is proved to fit within the framework of penalty-based aggregation in Section 4 . A discussion on the use of convex hull internal functions for the aggregation of multidimensional data in the context of the aggregation of compositional data is addressed in Section 5. We end with some conclusions in Section 6.

\section{Aggregation theory: State of the art}

Aggregation functions [5, 12] represent the core of one of the most important spin-offs of the fuzzy set community: aggregation theory.

Definition 1 Consider a bounded poset $(X, \leq, 0,1)$ and $n \in \mathbb{N}$. A function $A: X^{n} \rightarrow X$ is called an (n-ary) aggregation function on $(X, \leq, 0,1)$ if 
(i) it satisfies the boundary conditions, i.e., $A(0, \ldots, 0)=0$ and $A(1, \ldots, 1)=1$;

(ii) it is monotone, i.e., for any $\mathbf{x}, \mathbf{y} \in X^{n}$, the fact that $x_{i} \leq y_{i}$ for any $i \in\{1, \ldots, n\}$ implies that $A(\mathbf{x}) \leq A(\mathbf{y})$.

For historical reasons, and because they surely are the most natural examples of aggregation function, idempotent aggregation functions (also referred to as averaging functions or means) have been deeply studied by researchers coming from different fields [4, 13].

Definition 2 Consider a poset $(X, \leq)$ and $n \in \mathbb{N}$. A function $A: X^{n} \rightarrow X$ on $(X, \leq)$ is called an (n-ary) averaging function on $(X, \leq)$ if

(i) it it is idempotent, i.e., $A(x, \ldots, x)=x$, for any $x \in X$;

(ii) it is monotone.

Although both definitions above have been historically studied in the context of real numbers, in the last decades we are witnessing an increasing interest in the more generic framework of aggregation on (bounded) posets $[8,15]$.

A prominent family of averaging functions is that of penalty-based aggregation functions, which are built around the notion of a penalty function $[6,7,20]$. In this context, the property of monotonicity is oftentimes forfeited. Although the definition of a penaltybased aggregation function is typically restricted to the aggregation of real numbers, in a recent paper [18] we proposed a natural generalization of this type of function built upon the notion of a betweenness relation. Note that posets are probably the most common example of set equipped with a betweenness relation, but we can find more general examples such as the set of compositional data vectors (which will be discussed here in the upcoming sections).

Definition 3 A ternary relation $B$ on a non-empty set $X$ is called a betweenness relation if it satisfies the following three properties:

(i) Symmetry in the end points: for any $x, y, z \in X$, it holds that

$$
(x, y, z) \in B \Leftrightarrow(z, y, x) \in B .
$$

(ii) Closure: for any $x, y, z \in X$, it holds that

$$
((x, y, z) \in B \wedge(x, z, y) \in B) \Leftrightarrow y=z .
$$

(iii) End-point transitivity: for any $o, x, y, z \in X$, it holds that

$$
((o, x, y) \in B \wedge(o, y, z) \in B) \Rightarrow(o, x, z) \in B .
$$

A betweenness relation provides a set with some desirable semantics. Thus, we can construct a penalty function measuring the disagreement of a single object with a given list of objects to be aggregated that preserves the semantics provided by a given betweenness relation.

Definition 4 Consider $n \in \mathbb{N}$, a set $X$ and a betweenness relation $B$ on $X^{n}$. A function $P: X \times X^{n} \rightarrow \mathbb{R}^{+}$ is called a penalty function (compatible with $B$ ) if the following four properties hold:

(P1) $P(y ; \mathbf{x}) \geq 0$, for any $y \in X$ and any $\mathbf{x} \in X^{n}$;

(P2) $P(y ; \mathbf{x})=0$ if and only if $\mathbf{x}=(y, \ldots, y)$;

(P3) The set of minimizers of $P(\cdot ; \mathbf{x})$ is non-empty, for any $\mathbf{x} \in X^{n}$.

(P4) $P(y ; \mathbf{x}) \leq P\left(y ; \mathbf{x}^{\prime}\right)$, for any $y \in X$ and any $\mathbf{x}, \mathbf{x}^{\prime} \in$ $X^{n}$ such that $\left((y, \ldots, y), \mathbf{x}, \mathbf{x}^{\prime}\right) \in B$.

Remark 1 Two additional desirable properties for a penalty function are:

(P5) $P(y ; \mathbf{x}) \leq P\left(y^{\prime} ; \mathbf{x}\right)$, for any $y, y^{\prime} \in X$, any $\mathbf{x} \in X^{n}$ and any minimizer $z \in X$ of $P(\cdot ; \mathbf{x})$ such that $\left((z, \ldots, z),(y, \ldots, y),\left(y^{\prime}, \ldots, y^{\prime}\right)\right) \in B$

(P6) $P(y ; \mathbf{x})=P(z ; \mathbf{x})$, for any $y \in X$, any $\mathbf{x} \in X^{n}$ and any two minimizers $z, z^{\prime} \in X$ of $P(\cdot ; \mathbf{x})$ such that $\left((z, \ldots, z),(y, \ldots, y),\left(z^{\prime}, \ldots, z^{\prime}\right)\right) \in B$.

Ultimately, the penalty-based function associated with a penalty function is defined as the function that, given a list of objects to be aggregated, outputs the object that minimizes the disagreement with the given list of objects. If this minimizer is not unique, the function will output the set of minimizers. For this reason, we deal with functions of the type $f: X^{n} \rightarrow \mathcal{P}(X)$, where $\mathcal{P}(X)$ represents the powerset of $X$.

Definition 5 Consider $n \in \mathbb{N}$, a set $X$, a betweenness relation $B$ on $X^{n}$ and a penalty function $P: X \times X^{n} \rightarrow$ $\mathbb{R}^{+}$compatible with $B$. The function $f: X^{n} \rightarrow \mathcal{P}(X)$ defined by

$$
f(\mathbf{x})=\underset{y \in X}{\arg \min } P(y ; \mathbf{x}),
$$

for any $\mathbf{x} \in X^{n}$, is called the penalty-based function associated with $P$.

Note that this function is always idempotent but does not need to be monotone (there might be no associated order relation). However, it can be used for aggregating any type of objects, even in the absence of an associated order relation. 


\section{Aggregation of compositional data as the result of a mixture}

Vectors of positive real numbers adding up to one are referred to as compositional data vectors. Due to their natural interpretation as the proportions of different compounds in a mixture, they are common in many fields of application [1, 2], e.g., soil science, chemistry, biology and environmental science. The set of all $k$ dimensional compositional data vectors is referred to as the $k$-dimensional simplex and is defined as

$$
\mathcal{S}_{k}=\left\{\mathbf{x} \in[0,1]^{k} \mid \sum_{j=1}^{k} \mathbf{x}(j)=1\right\} .
$$

For the case $k=3$, the simplex can be naturally represented by an equilateral triangle where the length of each of the medians ${ }^{1}$ equals one. Every point inside the equilateral triangle corresponds to a point of the simplex. The coordinates of any point are obtained by the projection of the given point to each of the medians. As an illustrative example, we refer to Figure 1 for visualizing the coordinates of the compositional data vector $(0.55,0.32,0.13)^{T}$.

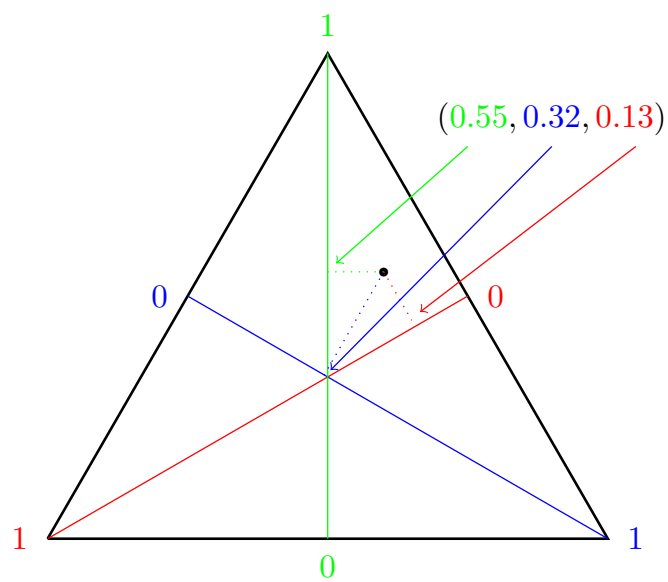

Figure 1: Graphical representation of the 3dimensional simplex.

When dealing with the aggregation of compositional data, the notion of monotonicity is somehow questionable since there is no notion of order and there is no compositional data vector that is greater than another (let aside the Lorenz order [17] and similar notions related to the inequality/diversity of the compositional data vectors which require a reordering of the compounds). However, one might still find the aggregation of compositional data in daily-life exercises, e.g., when mixing two liquids. Precisely, when mixing two liquids of known compositions in a one-to-one proportion, the composition of the resulting liquid is known

\footnotetext{
${ }^{1} \mathrm{~A}$ median of a triangle is a line segment joining a vertex to the midpoint of the opposite side.
}

to be given by the componentwise arithmetic mean (usually referred to as the centroid) of the compositions of both original liquids. For a different mixing ratio, the composition of the resulting liquid is then known to be given by the componentwise extension of a single weighted arithmetic mean (usually referred to as a weighted centroid).

Formally, we can aggregate $n$ compositional data vectors $\mathbf{x}_{1}, \ldots, \mathbf{x}_{n} \in \mathcal{S}_{k}$, resulting in a new compositional data vector, by using the function $\mathbf{C}_{\mathbf{w}}:\left(\mathcal{S}_{k}\right)^{n} \rightarrow \mathcal{S}_{k}$ defined as

$$
\mathbf{C}_{\mathbf{w}}\left(\mathbf{x}_{1}, \ldots, \mathbf{x}_{n}\right)(j)=\sum_{i=1}^{n} w_{i} \mathbf{x}_{i}(j),
$$

for any $j \in\{1, \ldots, k\}$, where $\mathbf{w}=\left(w_{1}, \ldots, w_{n}\right)$ is a suitable weighing vector such that $w_{i}>0$ for any $i \in\{1, \ldots, n\}$ and $\sum_{i=1}^{n} w_{i}=1$ (in the case of the liquids, the weighing vector represents the mixing ratio associated with each of the different liquids in the mixture).

Example 1 As an illustrative example, consider the compositional data vectors $\mathbf{x}_{1}=(0.55,0.32,0.13)^{T}$ and $\mathbf{x}_{2}=(0.03,0.72,0.25)^{T}$ representing the composition of two liquids in terms of three compounds. If we mix both liquids, in the same quantity, the resulting mixture will have the following composition:

$$
\mathbf{C}_{\left(\frac{1}{2}, \frac{1}{2}\right)}\left(\mathbf{x}_{1}, \mathbf{x}_{2}\right)=(0.29,0.52,0.19)^{T} .
$$

In case different quantities are used for each of the liquids, a weighted arithmetic mean, instead of the usual arithmetic mean, needs to be considered. For instance, in case we mix the previous two liquids in such a way that the quantity of $\mathbf{x}_{1}$ is the triple of the quantity of $\mathbf{x}_{2}$, the following compositional data vector would be obtained:

$$
\mathbf{C}_{\left(\frac{3}{4}, \frac{1}{4}\right)}\left(\mathbf{x}_{1}, \mathbf{x}_{2}\right)=(0.42,0.42,0.16)^{T} .
$$

As intuitive as this sounds, this function is not an aggregation function in the most classical sense. There is no intuitive order relation - and perhaps the property of monotonicity should be abandoned - but it is clear that this should be understood as an aggregation process. In the upcoming section we present the componentwise extension of a single weighted arithmetic mean within the framework of penalty-based aggregation.

\section{Penalty-based aggregation of compositional data}

In the context of compositional data there is no intuitive notion of order, however, there is indeed an 
intuitive notion of betweenness. This notion is captured by the betweenness relation $B_{\mathcal{S}_{k}}$ on $\mathcal{S}_{k}$, defined as follows:

$B_{\mathcal{S}_{k}}=\left\{\begin{array}{l|l}(\mathbf{x}, \mathbf{y}, \mathbf{z}) \in\left(\mathcal{S}_{k}\right)^{3} & \begin{array}{l}(\forall j \in\{1, \ldots, k\}) \\ (\min (\mathbf{x}(j), \mathbf{z}(j)) \leq \mathbf{y}(j) \leq \max (\mathbf{x}(j), \mathbf{z}(j)))\end{array}\end{array}\right.$.

This betweenness relation is illustrated in Figure 2 .

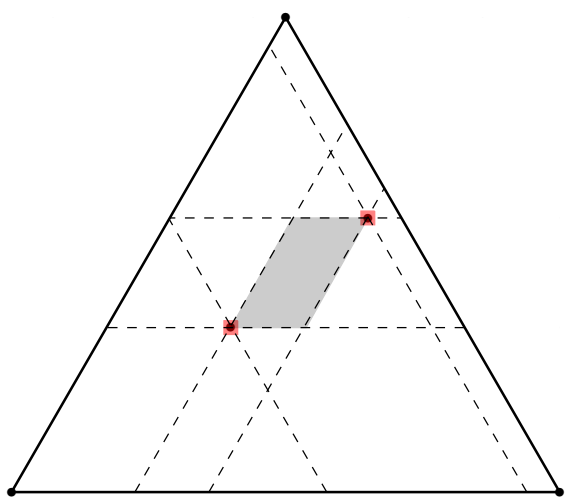

Figure 2: Illustration of the compositional data vectors (highlighted in grey) that are strictly in between the compositional data vectors that are highlighted in red according to the betweenness relation $B_{\mathcal{S}_{3}}$.

In case we are dealing with lists of compositional data vectors, we should consider the associated product betweenness relation, defined as:

$B_{\mathcal{S}_{k}}^{(n)}=\left\{\begin{array}{l}\left(\left(\mathbf{x}_{1}, \ldots, \mathbf{x}_{n}\right),\left(\mathbf{y}_{1}, \ldots, \mathbf{y}_{n}\right),\left(\mathbf{z}_{1}, \ldots, \mathbf{z}_{n}\right)\right) \in\left(\mathcal{S}_{k}^{n}\right)^{3} \\ \mid(\forall i \in\{1, \ldots, n\})\left(\left(\mathbf{x}_{i}, \mathbf{y}_{i}, \mathbf{z}_{i}\right) \in B_{\mathcal{S}_{k}}\right)\end{array}\right\}$.

In the following, we want to prove that any componentwise extension of a single weighted arithmetic mean is an example of penalty-based function compatible with the betweenness relation above.

Proposition 1 For any weighing vector $\mathbf{w}=$ $\left(w_{1}, \ldots, w_{n}\right\}$, the function $\mathbf{C}_{\mathbf{w}}:\left(\mathbb{R}^{k}\right)^{n} \rightarrow \mathbb{R}^{k}$ defined by $\mathbf{C}_{\mathbf{w}}\left(\mathbf{x}_{1}, \ldots, \mathbf{x}_{n}\right)(j)=\sum_{i=1}^{n} w_{i} \mathbf{x}_{i}(j)$, for any $j \in\{1, \ldots, k\}$, is a penalty-based function compatible with $B_{\mathcal{S}_{k}}^{(n)}$.

Proof. First, we can recall that any componentwise extension of a single weighted arithmetic mean could be characterized as follows:

$$
\begin{aligned}
\mathbf{C}_{\mathbf{w}}\left(\mathbf{x}_{1}, \ldots, \mathbf{x}_{n}\right) & =\underset{\mathbf{y}}{\arg \min } \sum_{i=1}^{n} w_{i} d\left(\mathbf{x}_{i}, \mathbf{y}\right)^{2} \\
& =\underset{\mathbf{y}}{\arg \min } \sum_{i=1}^{n} w_{i} \sum_{j=1}^{k}\left(\mathbf{x}_{i}(j)-\mathbf{y}(j)\right)^{2} .
\end{aligned}
$$

We could then consider the penalty function $P: \mathcal{S}_{k} \times$ $\mathcal{S}_{k}^{n} \rightarrow \mathbb{R}^{+}$defined as

$$
P\left(\mathbf{y},\left(\mathbf{x}_{1}, \ldots, \mathbf{x}_{n}\right)\right)=\sum_{i=1}^{n} w_{i} \sum_{j=1}^{k}\left(\mathbf{x}_{i}(j)-\mathbf{y}(j)\right)^{2} .
$$

We now prove that all properties for a penalty function compatible with $B_{\mathcal{S}_{k}}^{(n)}$ are fulfilled.

Both (P1) and (P2) follow from the fact that we are adding positive values and that each addend equals zero if and only if $\mathbf{x}_{i}(j)=\mathbf{y}(j)$. Furthermore, (P3) follows straightforwardly from the characterization of the (unique) minimizer of $P\left(\cdot ;\left(\mathbf{x}_{1}, \ldots, \mathbf{x}_{n}\right)\right)$ as the componentwise extension of the weighted arithmetic mean associated with the weight vector $\mathbf{w}$ of the compositional data vectors $\mathbf{x}_{1}, \ldots, \mathbf{x}_{n}$. Finally, (P4) follows from the fact that, given any $\mathbf{y} \in \mathcal{S}_{k}$ and any $\left(\mathbf{x}_{1}, \ldots, \mathbf{x}_{n}\right),\left(\mathbf{x}_{1}^{\prime}, \ldots, \mathbf{x}_{n}^{\prime}\right) \in \mathcal{S}_{k}^{n}$ such that $\left((\mathbf{y}, \ldots, \mathbf{y}),\left(\mathbf{x}_{1}, \ldots, \mathbf{x}_{n}\right),\left(\mathbf{x}_{1}^{\prime}, \ldots, \mathbf{x}_{n}^{\prime}\right)\right) \in B_{\mathcal{S}_{k}}^{(n)}$, it holds that

$$
\left(\mathbf{x}_{i}(j)-\mathbf{y}(j)\right)^{2} \leq\left(\mathbf{x}_{i}^{\prime}(j)-\mathbf{y}(j)\right)^{2},
$$

for any $i \in\{1, \ldots, n\}$ and any $j \in\{1, \ldots, k\}$.

The additional desirable properties are also satisfied. Since there exists a unique minimizer of $P\left(\cdot ;\left(\mathbf{x}_{1}, \ldots, \mathbf{x}_{n}\right)\right)$ for any $\left(\mathbf{x}_{1}, \ldots, \mathbf{x}_{n}\right) \in \mathcal{S}_{k}^{n},(\mathrm{P} 6)$ is trivially satisfied. Finally, (P5) follows from the fact that, given any $\mathbf{y}, \mathbf{y}^{\prime} \in \mathcal{S}_{k}$ and any $\left(\mathbf{x}_{1}, \ldots, \mathbf{x}_{n}\right) \in \mathcal{S}_{k}^{n}$ such that $\left((\mathbf{y}, \ldots, \mathbf{y}),\left(\mathbf{y}^{\prime}, \ldots, \mathbf{y}^{\prime}\right),(\mathbf{z}, \ldots, \mathbf{z})\right) \in B_{\mathcal{S}_{k}}^{(n)}$ (where $\mathbf{z}=\mathbf{C}_{\mathbf{w}}\left(\mathbf{x}_{1}, \ldots, \mathbf{x}_{n}\right)$ is the unique minimizer of $\left.P\left(\cdot ;\left(\mathbf{x}_{1}, \ldots, \mathbf{x}_{n}\right)\right)\right)$, it holds that

$$
\min (\mathbf{y}(j), \mathbf{z}(j)) \leq \mathbf{y}^{\prime}(j) \leq \max (\mathbf{y}(j), \mathbf{z}(j)),
$$

for any $j \in\{1, \ldots, k\}$, and, thus, due to the convexity of the function $F(t)=\sum_{i=1}^{n} w_{i}\left(\mathbf{x}_{i}(j)-t\right)^{2}$, it also holds that

$$
\begin{aligned}
F(\mathbf{y}(j)) & =\sum_{i=1}^{n} w_{i}\left(\mathbf{x}_{i}(j)-\mathbf{y}(j)\right)^{2} \\
& \leq \sum_{i=1}^{n} w_{i}\left(\mathbf{x}_{i}(j)-\mathbf{y}^{\prime}(j)\right)^{2} \\
& =F\left(\mathbf{y}^{\prime}(j)\right)
\end{aligned}
$$

for any $j \in\{1, \ldots, k\}$.

\section{Convex hull internal functions for aggregating multidimensional data}

In the field of (computational) statistics, there is a large literature on how to combine several (multidimensional) real vectors into a single one. For instance, the aforementioned componentwise extension of a single weighted arithmetic mean has been deeply analysed under the name of weighted centroid [11]. Great interest has also been devoted to the proposal of different generalizations of the (unidimensional) median in order to deal with (multidimensional) real vectors [19], highlighting the spatial median (also referred to as geometric median or Fermat-Weber point). 
An interesting property for such functions aiming at combining several (multidimensional) real vectors into a single one is that of convex hull internality [10]. This property requires the result of aggregating the (multidimensional) real vectors $\mathbf{x}_{1}, \ldots, \mathbf{x}_{n} \in \mathbb{R}^{k}$ to belong to their convex hull, i.e., the set

$$
\left\{\mathbf{x}=\sum_{i=1}^{n} \lambda_{i} \mathbf{x}_{i} \in \mathbb{R}^{k} \mid\left(\lambda_{1}, \ldots, \lambda_{n}\right) \in \mathcal{S}_{n}\right\} .
$$

Typical examples of convex hull internal functions are the aforementioned weighted centroid and spatial median. Some other generalizations of the unidimensional median are also known to be convex hull internal (e.g., the convex hull stripping median [9]), whereas some others are known to fail this intuitive property (e.g., the componentwise median).

This property is of key importance to the aggregation of compositional data. Precisely, all convex hull internal functions for the aggregation of multidimensional data can be used for the aggregation of compositional data, even though - unlike with the weighted centroid - an intuitive physical meaning could be lacking.

Proposition 2 If a function $\mathbf{A}:\left(\mathbb{R}^{k}\right)^{n} \rightarrow \mathbb{R}^{k}$ is convex hull internal, then, for any $\left(\mathbf{x}_{1}, \ldots, \mathbf{x}_{n}\right) \in\left(\mathcal{S}_{k}\right)^{n}$, it holds that $\mathbf{A}\left(\mathbf{x}_{1}, \ldots, \mathbf{x}_{n}\right) \in \mathcal{S}_{k}$.

Proof. Consider $\left(\mathbf{x}_{1}, \ldots, \mathbf{x}_{n}\right) \in\left(\mathcal{S}_{k}\right)^{n}$. Since $\mathbf{A}:\left(\mathbb{R}^{k}\right)^{n} \rightarrow \mathbb{R}^{k}$ is convex hull internal, it follows that $\mathbf{A}\left(\mathbf{x}_{1}, \ldots, \mathbf{x}_{n}\right)$ belongs to the convex hull of $\mathbf{x}_{1}, \ldots, \mathbf{x}_{n}$. Since all $\mathbf{x}_{1}, \ldots, \mathbf{x}_{n}$ belong to $\mathcal{S}_{k}$, the convex hull of $\mathbf{x}_{1}, \ldots, \mathbf{x}_{n}$ needs to be a subset of the convex hull of $\mathcal{S}_{k}$. Finally, since $\mathcal{S}_{k}$ is already a convex set, it coincides with its convex hull and, thus, we conclude that $\mathbf{A}\left(\mathbf{x}_{1}, \ldots, \mathbf{x}_{n}\right) \in \mathcal{S}_{k}$.

We end the section by noting that some convex hull internal functions for aggregating multidimensional data (e.g. the spatial median) are easily proven to fit within the penalty-based aggregation framework discussed in Section 2, whereas some others (e.g. the convex hull stripping median) do not fit within said framework.

\section{Conclusions}

The field of aggregation theory has historically built upon the property of monotonicity. In a recent paper, Gagolewski [10] raises the question on whether this monotonicity property should be sometimes forfeited in the setting of multidimensional data, for which the poset structure is possibly questionable. In particular, some further results by Gagolewski and the present authors [11] prove that when idempotence is combined with equivariance to orthogonal transformations (which is a classical property in multivariate statistics), the only monotone functions that remain are componentwise extensions of a single weighted arithmetic mean (or weighted centroids).

In this conference paper, we went further and discussed the setting of compositional data in which the property of monotonicity is not only "questionable" but actually nonsensical. The weighted centroid, which in the setting of compositional data represents the result of a mixing process, was proved to fit within the framework of penalty-based aggregation introduced in [18]. A final discussion on the use of convex hull internal functions for the aggregation of multidimensional data in the context of the aggregation of compositional data opens several future research topics. A further study of potential definitions of betweenness-based monotonicity properties for these functions is yet to be addressed.

\section{Acknowledgement}

Raúl Pérez-Fernández acknowledges the support of the Research Foundation of Flanders (FWO17/PDO/160) and the Spanish MINECO (TIN2017-87600-P).

\section{References}

[1] J. Aitchison, The statistical analysis of compositional data (with discussion), Journal of the Royal Statistical Society B44 (1982) 139-177.

[2] J. Aitchison, The Statistical Analysis of Compositional Data, Blackburn Press, 2003.

[3] J. P. Barthelemy, B. Monjardet, The median procedure in cluster analysis and social choice theory, Mathematical Social Sciences 1 (1981) 235-267.

[4] G. Beliakov, H. Bustince, T. Calvo, A Practical Guide to Averaging Functions, Studies in Fuzziness and Soft Computing, Springer-Verlag, Berlin, Heidelberg, Berlin, 2016.

[5] G. Beliakov, A. Pradera, T. Calvo, Aggregation Functions: A Guide for Practitioners, Vol. 221 of Studies in Fuzziness and Soft Computing, Springer, Berlin, Heidelberg, 2007.

[6] H. Bustince, G. Beliakov, G. P. Dimuro, B. Bedregal, R. Mesiar, On the definition of penalty functions in data aggregation, Fuzzy Sets and Systems 323 (2017) 1-18.

[7] T. Calvo, G. Beliakov, Aggregation functions based on penalties, Fuzzy Sets and Systems 161 (2010) 1420-1436.

[8] D. Dubois, H. Prade, On the use of aggregation operations in information fusion processes, Fuzzy Sets and Systems 142 (2004) 143-161.

[9] W. F. Eddy, Convex hull peeling, in: COMPSTAT Symposium, Toulouse, 1982, pp. 42-47. 
[10] M. Gagolewski, Penalty-based aggregation of multidimensional data, Fuzzy Sets and Systems 325 (2017) 4-20.

[11] M. Gagolewski, R. Pérez-Fernández, B. De Baets, An inherent difficulty in the aggregation of multidimensional data, submitted.

[12] M. Grabisch, J.-L. Marichal, R. Mesiar, E. Pap, Aggregation Functions, Cambridge University Press, Cambridge, 2009.

[13] M. Grabisch, J.-L. Marichal, R. Mesiar, E. Pap, Aggregation functions: Means, Information Sciences 181 (2011) 1-22.

[14] J. G. Kemeny, Mathematics without numbers, Daedalus 88 (4) (1959) 577-591.

[15] M. Komorníková, R. Mesiar, Aggregation functions on bounded partially ordered sets and their classification, Fuzzy Sets and Systems 175 (2011) $48-56$.

[16] J. K. Lanctot, M. Li, B. Ma, S. Wang, L. Zhang, Distinguishing string selection problems, Information and Computation 185 (2003) 41-55.

[17] M. O. Lorenz, Methods of measuring the concentration of wealth, Publications of the American Statistical Association 9 (70) (1905) 209-219.

[18] R. Pérez-Fernández, B. De Baets, On the role of monometrics in penalty-based data aggregation, IEEE Transactions on Fuzzy Systems, in press, DOI: $10.1109 /$ TFUZZ.2018.2880716.

[19] C. G. Small, A survey of multidimensional medians, International Statistical Review 58 (3) (1990) 263-277.

[20] R. R. Yager, Toward a general theory of information aggregation, Information Sciences 68 (1993) 191-206. 